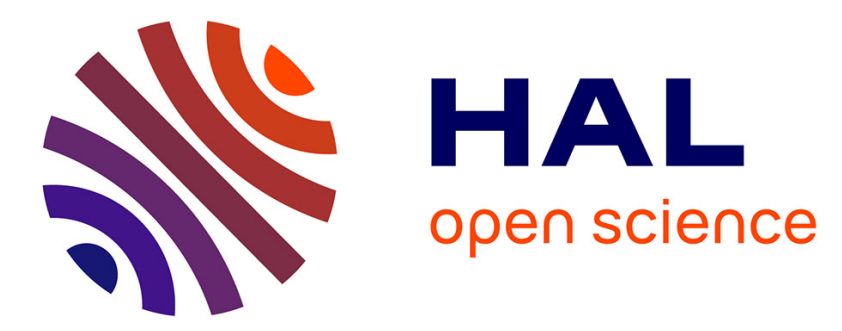

\title{
Adaptive observers-based synchronization of a class of Lur'e systems with delayed outputs for chaotic communications
}

\author{
Habib Dimassi, Antonio Loria, Safya Belghith
}

\section{- To cite this version:}

Habib Dimassi, Antonio Loria, Safya Belghith. Adaptive observers-based synchronization of a class of Lur'e systems with delayed outputs for chaotic communications. CHAOS, Jun 2012, Mexico. pp.255260, 10.3182/20120620-3-MX-3012.00015 . hal-00831383

\section{HAL Id: hal-00831383 \\ https://hal.science/hal-00831383}

Submitted on 6 Jun 2013

HAL is a multi-disciplinary open access archive for the deposit and dissemination of scientific research documents, whether they are published or not. The documents may come from teaching and research institutions in France or abroad, or from public or private research centers.
L'archive ouverte pluridisciplinaire HAL, est destinée au dépôt et à la diffusion de documents scientifiques de niveau recherche, publiés ou non, émanant des établissements d'enseignement et de recherche français ou étrangers, des laboratoires publics ou privés. 


\title{
Adaptive observers-based synchronization of a class of Lur'e systems with delayed outputs for chaotic communications
}

\author{
H. Dimassi* A. Loria ${ }^{* *}$ S. Belghith ${ }^{* * *}$ \\ * CNRS, LSS-Supelec, Univ Paris Sud and Sys' com-Ecole Nationale \\ d'Ingénieurs de Tunis, Tunisia. E-mail: dimassi@lss.suepelc.fr \\ ** CNRS, LSS-Supelec, 3 Rue Joliot Curie, Gif sur Yvette France. \\ E-mail: loria@lss.suepelc.fr \\ *** Sys' com-Ecole Nationale d'Ingénieurs de Tunis, Tunisia. E-mail: \\ safya.belghith@enit.rnu.tn
}

\begin{abstract}
In this paper, we propose an adaptive observer-based synchronization approach for a class of chaotic Lur'e systems with slope restricted nonlinearities and delayed outputs. The delay is assumed bounded and time varying and the information to be transmitted is assumed piece-wise constant. Based on the Lyapunov-Krasovskii approach, we show that for sufficiently small values of the time-delay upper bound, both synchronization and information reconstruction objectives are ensured under a condition of persistent excitation and after solving a convex optimization problem. The result is illustrated via a numerical example of a chaotic communication system subject to a transmission delay.
\end{abstract}

Keywords: Chaos, synchronization, communications, time delay, state observers, adaptive control

\section{INTRODUCTION}

The problem of synchronization of chaotic Lur'e systems with a propagation delay has attracted specific attention in the last decade. Indeed, time delays are unavoidable in practice and occur frequently in master-slave synchronization configurations and their applications in chaotic transmission schemes. To solve this problem, a common approach in the literature is to use error state or/and output feedback controllers and exploit sector and slope restrictions properties of Lur'e systems - see Yalcin et al. (2001), Cao et al. (2005), Han (2007a), Han (2007b), Lee et al. (2010).

The first work investigating this problem was done in the reference Yalcin et al. (2001) in which a delay dependent synchronization criterion was given based on a Lyapunov-Krasovskii functional. This result has motivated many researches on the synchronization of chaotic Lur'e systems using feedback controllers. In Cao et al. (2005), both delay dependent and delay independent criteria for synchronization are given. In Han (2007a), the proposed feedback controller includes both the current error state feedback and the delayed static error output feedback; and based on a more general Lur'e-Postnikov Lyapunov functional, a new delay dependent criteria are presented in the form of linear matrix inequalities (LMIs). This problem was extended in Han (2007b) for the case of continuous uniformly bounded time varying delays and differentiable uniformly bounded time varying delays with bounded derivatives. More recently, a less conservative delay dependent synchronization criterion was obtained in Lee et al. (2010) using a new Lyapunov-Krasovskii functional which employs redundant state of differential equations shifted in time by a fraction of the time delay.

On the other hand, the problem of observation of nonlinear systems in the case of delayed outputs was investigated in the last decade. In the reference Germani et al. (2000), based on the design of state observers from a driftobservability property, the authors propose a chain of observation algorithms which ensure global exponential convergence of the estimation error. In a recent work Cacace et al. (2010), the authors propose a nonlinear observer for a class of drift observable nonlinear systems with a bounded time varying observation delay. Based on the Lyapunov-Razumikhin approach, asymptotic and exponential convergence of the estimation error have been proved to be guaranteed under some conditions. Another predictor based on high gain observer design for a class of triangular nonlinear systems with respectively constant and time varying delayed measurements was given in Ahmed-Ali et al. (2009) and Assche et al. (2011); the convergence was shown to be ensured by choosing a suitable Lyapunov-Krasovskii functional if some condition relating the observer gain and the upper bound of the time delay is satisfied.

In Nijmeijer and Mareels (1997), it was shown that the master-slave synchronization problem may be considered from a control viewpoint in a paradigm of observer design where the slave system is considered as an observer of the master system. A particular problem of observer design is to join state and unknown parameters estimation using adaptive observers. The recent reference Loria et al. (2009) investigates this problem in the context of synchroniza- 
tion of chaotic systems with parametric uncertainties by exploiting the property of persistent excitation. Adaptive observer-based synchronization methods were employed in chaotic communication schemes to transmit digital data using the chaotic modulation method based on modulating one of the master system parameters by the encoded information -see Dimassi and Loria (2011), Feki (2003).

In the present paper, we propose an adaptive observerbased synchronization approach for a class of chaotic Lur'e systems with slope restricted nonlinearities and delayed outputs. The paper is organized as follows. In the following section, we motivate and formulate the problem. In Section 3 , we present our approach and the stability analysis. In Section 4, we illustrate our results via a numerical example of a chaotic transmission application. Finally, some concluding remarks will be given in Section 5 .

Notation. In this paper, $|\cdot|$ denotes the absolute value for scalars, the Euclidean norm for vectors, and the induced norm for matrices. $I$ represents the identity matrix. The smallest and largest eigenvalues of $S$ are denoted by $\lambda_{\min }(S)$ and $\lambda_{\max }(S)$ respectively.

\section{PROBLEM STATEMENT}

We consider a chaotic communication system consisting of a transmitter and a receiver. Taking into account the presence of a transmission delay in the communication channel, the transmitter is based on a class of chaotic Lur'e systems and represented as follows

$$
\begin{aligned}
& \dot{x}(t)=A x(t)+F f(H x(t))+B \Psi(t) \theta(t) \\
& y(t)=C x(t-h(t))
\end{aligned}
$$

where $x \in \mathbb{R}^{n}$ denotes the state vector; $y \in \mathbb{R}^{p}$ represents the output vector (subject to the transmission delay $h(t)$ ). It is assumed that the delay $h(t)$ is a knwon time varying function satisfying $0 \leq h(t) \leq h_{\max }, \forall t \geq 0 . A \in$ $\mathbb{R}^{n \times n}, B \in \mathbb{R}^{n \times s}, F \in \mathbb{R}^{n \times m}, C \in \mathbb{R}^{p \times n}$ and $H=$ $\left(H_{1}, H_{2}, \ldots, H_{m}\right)^{T} \in \mathbb{R}^{m \times n}$ are constant matrices; $H_{i}$ is the $i-t h$ raw of $H ; i \in\{1,2, \ldots, m\}$. The function $\theta(t)$ : $\mathbb{R}_{\geq 0} \rightarrow \mathbb{R}^{q}$ represents the vector of the information signal to be transmitted. We assume $\theta(t)$ piece-wise constant. $\Psi(t): \mathbb{R}_{+} \rightarrow \mathbb{R}^{s \times q}$ is a persistently exciting function. It is assumed that $\exists \Psi_{\max }>0$ such that

$$
\sup _{t \geq 0}|\Psi(t)| \leq \Psi_{\max }
$$

Remark 1. The assumption that the time-delay is known in real-time which is frequently used in the literature may be restrictive from a practical view point. It means that at the actual time $t$, the output $y(t)$ and the instant $t_{r}$ at which the signal arrives at the receiver end are assumed known, hence the delay may be calculated as $h(t)=t-t_{r}$.

$f(\cdot): \mathbb{R}^{m} \rightarrow \mathbb{R}^{m}$ is a nonlinear function where

$f(H x)=\left(f_{1}\left(H_{1} x\right), f_{2}\left(H_{2} x\right), \ldots, f_{m}\left(H_{m} x\right)\right)$;

we assume that $\forall i \in\{1,2, \ldots, m\}, f_{i}(\cdot)$ satisfies the following slope restriction condition,

$\forall \xi_{1}, \xi_{2} \in \mathbb{R}$ such that $\xi_{1} \neq \xi_{2}$, we have

$$
0 \leq \frac{f_{i}\left(\xi_{1}\right)-f_{i}\left(\xi_{2}\right)}{\xi_{1}-\xi_{2}} \leq b
$$

Remark 2. If instead of the condition $(3), f_{i}(\cdot)$ satisfies $\forall \xi_{1}, \xi_{2} \in \mathbb{R}$ such that $\xi_{1} \neq \xi_{2}$, we have

$$
a_{i} \leq \frac{f_{i}\left(\xi_{1}\right)-f_{i}\left(\xi_{2}\right)}{\xi_{1}-\xi_{2}} \leq b_{i},
$$

by considering the function $\tilde{f}_{i}(\xi):=f_{i}(\xi)-a_{i} \xi$, it is not difficult to show that $\tilde{f}_{i}(\cdot)$ satisfies the slope restriction (3) with $b=\max \left\{b_{i}-a_{i}, i=1,2, \ldots, m\right\}$, and rewrite the system (1) as

$$
\begin{aligned}
\dot{x}(t) & =\tilde{A} x(t)+F \tilde{f}(H x(t))+B \Psi(t) \theta(t) \\
y(t) & =C x(t-h(t)),
\end{aligned}
$$

with $\tilde{f}(H x)=\left(\tilde{f}_{1}\left(H_{1} x\right), \tilde{f}_{2}\left(H_{2} x\right), \ldots, \tilde{f}_{m}\left(H_{m} x\right)\right)$ and $\tilde{A}=A+F A_{d} H$, where $A_{d}=\operatorname{diag}\left(a_{1}, a_{2}, \ldots, a_{m}\right)$.

On the other side, if $f_{i}(\cdot)$ is continuously differentiable such that $a_{i} \leq \frac{\partial f_{i}(w)}{\partial w} \leq b_{i}, \forall w \in \mathbb{R}$, then applying the mean value theorem, $f_{i}(\cdot)$ satisfies the slope restriction (4). In this manner, the class of chaotic Lur'e systems that we consider in this paper is enlarged.

The objective of this paper is to design a slave system (the receiver) which synchronizes with the master system (1) and reconstructs the information signal $\theta(t)$ despite the time varying transmission delay $h(t)$.

\section{MAIN RESULT}

The slave system that we propose is designed as an adaptive observer described by the following equations

$$
\begin{aligned}
\dot{z}(t)= & A z(t)+F f(H z(t))+B \Psi(t) \hat{\theta}(t) \\
& +K(y(t)-C z(t-h(t))) \\
\dot{\hat{\theta}}(t)= & \rho \Upsilon(t)^{-1} \Psi(t)^{T} M(y(t)-C z(t-h(t))) \\
\dot{\Upsilon}(t)= & -\alpha \Upsilon(t)+\Psi(t)^{T} B^{T} B \Psi(t) \\
\Upsilon(0)= & \Upsilon(0)^{T}>0 .
\end{aligned}
$$

where $z(t)$ and $\hat{\theta}(t)$ denote respectively the estimated state and the recovered information vectors; $\rho$ and $\alpha$ are positive constants.

We define the synchronization error $e(t):=x(t)-z(t)$ and the adaptation error $\tilde{\theta}(t):=\theta(t)-\hat{\theta}(t)$.

Let $e_{h}(t):=e(t-h(t))$. Using the fact that $\dot{\theta}(t)=0$ almost everywhere, the error system is described by

$$
\begin{aligned}
\dot{e}(t)= & A e(t)-K C e_{h}(t)+F \eta(H e(t), x(t)) \\
& +B \Psi(t) \tilde{\theta}(t) \\
\dot{\tilde{\theta}}(t)= & -\rho \Upsilon(t)^{-1} \Psi(t)^{T} M C e_{h}(t) \quad \text { a.e; }
\end{aligned}
$$

where

$\eta(H e(t), x(t))=\left(\eta_{1}\left(H_{1} e(t), x(t)\right), \ldots, \eta_{m}\left(H_{m} e(t), x(t)\right)\right)$ and $\eta_{i}\left(H_{i} e(t), x(t)\right)=f_{i}\left(H_{i} x(t)\right)-f_{i}\left(H_{i} x(t)-H_{i} e(t)\right)$; $i \in\{1,2, \ldots, m\}$. Now, using the slope restriction (3) with $\xi_{1}=H_{i} x(t)$ and $\xi_{2}=H_{i} x(t)-H_{i} e(t)$, we have $\forall t \geq 0, \forall e(t) \neq 0, \forall x(t)$

$$
0 \leq \frac{\eta_{i}\left(H_{i} e(t), x(t)\right)}{H_{i} e(t)} \leq b
$$

Multiplying on both sides of (8) by $\eta_{i}\left(H_{i} e(t), x(t)\right) H_{i} e(t)$, we get:

for $i \in\{1,2, \ldots, m\}, \forall t \geq 0, \forall e(t), \forall x(t)$

$$
\eta_{i}\left(H_{i} e(t), x(t)\right)\left[\eta_{i}\left(H_{i} e(t), x(t)\right)-b H_{i} e(t)\right] \leq 0
$$


which means that $\eta(H e(t), x(t))$ belongs to the sector $[0, \mathrm{~b}]$. Let $D=\operatorname{diag}\left(d_{1}, d_{2}, \ldots, d_{m}\right)$ where $d_{i}>0 ; \forall i \in$ $\{1,2, \ldots, m\}$. After some manipulations, one can easily obtain the relation

$$
\begin{gathered}
-\eta^{T}(H e(t), x(t)) D \eta(H e(t), x(t)) \\
+b \eta(H e(t), x(t)) D H e(t) \geq 0
\end{gathered}
$$

Assumption 1. We assume that there exist a symmetric positive definite matrix $P$, a diagonal positive matrix $D$; regular matrices $M$ and $K$ of appropriate dimensions and a positive constant $\varepsilon$ such that

$$
\begin{gathered}
{\left[\begin{array}{cc}
-Q+\varepsilon I & P F+b H^{T} D \\
F^{T} P+b D H & -2 D
\end{array}\right] \leq 0} \\
B^{T} P=M C
\end{gathered}
$$

where $Q=-\left[(A-K C)^{T} P+P(A-K C)\right]$

Assumption 2. The matrix function $B \Psi(t)$ is persistently exciting in the sense that there exist $\mu, T>0$, such that

$$
\int_{t}^{t+T} \Psi(s)^{T} B^{T} B \Psi(s) d s \geq \mu I \quad \forall t \geq 0 .
$$

Remark 3. To solve (11), we consider the following convex optimization problem:

To minimize $\varsigma$ subject to

$$
\begin{gathered}
P>0 \\
D>0 \\
\varepsilon>0 \\
{\left[\begin{array}{cc}
\Xi & P F+b H^{T} D \\
F^{T} P+b D H & -2 D
\end{array}\right] \leq 0} \\
{\left[\begin{array}{cc}
\varsigma I & B^{\top} P-M C \\
P B-C^{\top} M^{\top} & \varsigma I
\end{array}\right] \leq 0,}
\end{gathered}
$$

where $\Xi=P A+A^{\top} P+W C_{1}+C^{\top} W^{\top}+\varepsilon I$.

The solution to this problem yields the minimum $\varsigma=0, \varepsilon$, $P, D, M$ and $W$ such that $K=-P^{-1} W$ satisfies (11a).

The following two Lemmas are required in the proof of our main result.

Lemma 1. Let $\Upsilon(t)$ the solution of the equation (6c). If $B \Psi(t)$ satisfies the assumption 2 , then $\Upsilon(t)$ is a symmetric positive definite matrix function such that

$$
\forall t \geq t_{0}+T, \Upsilon(t) \geq \mu e^{-\alpha T} I .
$$

Proof. The proof of Lemma 1 is provided in the Appendix.

Lemma 2. (Jensen's Inequality) (Pan (2008))

For any constant matrix $E \in \mathbb{R}^{n \times n}, E=E^{T}>0$, a vector function $w:[0, \tau] \rightarrow \mathbb{R}^{n}$ such that the integrations are well defined, then

$$
\tau \int_{0}^{\tau} w^{T}(s) E w(s) d s \geq\left(\int_{0}^{\tau} w(s) d s\right)^{T} E \int_{0}^{\tau} w(s) d s
$$

Now, we are ready to present our main result.

Theorem 1. Consider the master system (1) and the slave system (6). Let the assumptions 1 and 2 hold. Then, the origin $(e, \tilde{\theta})=(0,0)$ of the error system (7) is globally asymptotically stable for sufficiently small value of the time-delay upper bound $h_{\max }$.

Proof. Let us apply the Leibniz-Newton formula to the synchronization error $e(t)$. That is

$$
e(t)-e_{h}(t)=\int_{t-h(t)}^{t} \dot{e}(s) d s .
$$

Then, the dynamics of the synchronization error $e(t)$ can be rewritten as follows

$$
\begin{aligned}
\dot{e}(t)= & A e(t)-K C e(t)+F \eta(H e(t), x(t)) \\
& +B \Psi(t) \tilde{\theta}(t)+K C \int_{t-h(t)}^{t} \dot{e}(s) d s .
\end{aligned}
$$

Next, consider the Lyapunov-Krasovskii functional:

$$
\begin{aligned}
V(t, e, \tilde{\theta}) & =V_{1}(e)+V_{2}(t)+V_{3}(t, \tilde{\theta}), \\
V_{1}(e) & =e^{T} P e, \\
V_{2}(t) & =\int_{t-h_{\max }}^{t}\left(s-t+h_{\max }\right) \dot{e}(s)^{2} d s, \\
V_{3}(t, \tilde{\theta}) & =\rho^{-1} \tilde{\theta}^{T} \Upsilon(t) \tilde{\theta},
\end{aligned}
$$

where $\Upsilon(t)$ is governed by Equation (6c). One notices also that $\Upsilon(t)$ is bounded since $\Psi(t)$ is bounded. Let us define:

$$
\begin{aligned}
\Gamma(t) & :=\int_{t-h(t)}^{t} \dot{e}(s) d s ; \beta_{a}:=|A| ; \\
\beta_{b} & :=|B| \quad ; \beta_{c}:=|K C| .
\end{aligned}
$$

The time derivative of $V_{1}$ is given by

$$
\begin{aligned}
\dot{V}_{1}(e)= & e^{T}\left[(A-K C)^{T} P+P(A-K C)\right] e \\
& +2 e^{T} P F \eta(H e(t), x(t)) \\
& +2 e^{T} P K C \int_{t-h}^{t} \dot{e}(s) d s+2 e^{T} P B \Psi \tilde{\theta} .
\end{aligned}
$$

Using (10), we have

$$
\begin{aligned}
\dot{V}_{1}(e) \leq & e^{T}\left[(A-K C)^{T} P+P(A-K C)\right] e \\
& +2 e^{T} P F \eta(H e(t), x(t)) \\
& -2 \eta^{T}(H e(t), x(t)) D \eta(H e(t), x(t)) \\
& +2 b \eta(H e(t), x(t)) D H e(t) \\
& +2 e^{T} P K C \int_{t-h}^{t} \dot{e}(s) d s+2 e^{T} P B \Psi \tilde{\theta} .
\end{aligned}
$$

Let $\zeta(t):=(e(t), \eta(H e(t), x(t)))^{T}$. Using the expression of $Q$ defined above in assumption 1, then one has

$$
\begin{aligned}
\dot{V}_{1}(e) \leq & \zeta(t)^{T}\left[\begin{array}{cc}
-Q+\varepsilon I & P F+b H^{T} D \\
F^{T} P+b D H & -2 D
\end{array}\right] \zeta(t) \\
& +2 e^{T} P K C \int_{t-h}^{t} \dot{e}(s) d s \\
& +2 e^{T} C^{T} M^{T} \Psi \tilde{\theta}-\varepsilon|e|^{2},
\end{aligned}
$$

where we have also used (11b). After Assumption 1, we obtain 


$$
\begin{aligned}
\dot{V}_{1}(e) \leq & 2 e^{T} P K C \int_{t-h}^{t} \dot{e}(s) d s \\
& +2 e^{T} C^{T} M^{T} \Psi \tilde{\theta}-\varepsilon|e|^{2} .
\end{aligned}
$$

Applying the triangle inequality $\left|2 c^{T} d\right| \leq \gamma c^{T} c+\frac{1}{\gamma} d^{T} d$ to the term " $2 e(t)^{T} P K C \dot{e}(s)$ " with $c^{T}=e(t)^{T} P K C$, $d=\dot{e}(s)$ and $\gamma=2$, and integrating from $s=t-h(t)$ to $s=t$, we obtain

$$
\begin{aligned}
& 2 e(t)^{T} P K C \int_{t-h}^{t} \dot{e}(s) d s \\
\leq & 2 h(t) e(t)^{T} C^{T} K^{T} P^{2} K C e(t)+\frac{1}{2} \int_{t-h}^{t} \dot{e}(s)^{2} d s \\
\leq & 2 h_{\max } \beta_{c}^{2} \lambda_{\max }(P)^{2}|e(t)|^{2}+\frac{1}{2} \int_{t-h_{\max }}^{t} \dot{e}(s)^{2} d s
\end{aligned}
$$

hence,

$$
\begin{aligned}
\dot{V}_{1}(e) \leq & \left(-\frac{\varepsilon}{\lambda_{\max }(P)}+2 h_{\max } \beta_{c}^{2} \lambda_{\max }(P)^{2}\left(\lambda_{\min }(P)\right)^{-1}\right) V_{1}(e) \\
& +\frac{1}{2} \int_{t-h_{\max }}^{t} \dot{e}(s)^{2} d s+2 e^{T} C^{T} M^{T} \Psi \tilde{\theta}
\end{aligned}
$$

The time derivative of $V_{3}$ is given by

$$
\dot{V}_{3}(t, \tilde{\theta})=\dot{\tilde{\theta}}^{T} \Upsilon \tilde{\theta}+\tilde{\theta}^{T} \Upsilon \dot{\tilde{\theta}}+\tilde{\theta}^{T} \dot{\Upsilon} \tilde{\theta}
$$

Using (6c) and (7b), one obtains

$$
\begin{aligned}
\dot{V}_{3}(t, \tilde{\theta})= & -2 e_{h}^{T} C^{T} M^{T} \Psi \tilde{\theta}-\alpha \rho^{-1} \tilde{\theta}^{T} \Upsilon \tilde{\theta} \\
& +\rho^{-1} \tilde{\theta}^{T} \Psi^{T} B^{T} B \Psi \tilde{\theta}, \quad \text { a.e. }
\end{aligned}
$$

and after (20),

$$
\begin{aligned}
\dot{V}_{3}(t, \tilde{\theta})= & -2 e^{T} C^{T} M^{T} \Psi \tilde{\theta}+2 \Gamma^{T} C^{T} M^{T} \Psi \tilde{\theta} \\
& +\rho^{-1} \tilde{\theta}^{T} \Psi^{T} B^{T} B \Psi \tilde{\theta}-\alpha \rho^{-1} \tilde{\theta}^{T} \Upsilon \tilde{\theta} \quad \text { a.e. }
\end{aligned}
$$

Now, using the triangle inequality and Equation (11b), we obtain

$$
2 \Gamma^{T} C^{T} M^{T} \Psi \tilde{\theta} \leq \Gamma^{2}+\left(\Psi_{\max } \beta_{b} \lambda_{\max }(P)\right)^{2} \tilde{\theta}^{2},
$$

hence,

$$
\begin{aligned}
\dot{V}_{3}(t, \tilde{\theta}) \leq & -2 e^{T} C^{T} M^{T} \Psi \tilde{\theta}+\left(\Psi_{\max } \beta_{b} \lambda_{\max }(P)\right)^{2} \tilde{\theta}^{2} \\
& +\rho^{-1} \beta_{b}^{2} \Psi_{\max }^{2} \tilde{\theta}^{2}+\Gamma^{2}-\alpha \rho^{-1} \tilde{\theta}^{T} \Upsilon \tilde{\theta}
\end{aligned}
$$

Using Lemma 1, it follows that

$$
\begin{gathered}
\dot{V}_{3}(t, \tilde{\theta}) \leq \\
-\alpha V_{3}-2 e^{T} C^{T} M^{T} \Psi \tilde{\theta}+\Gamma^{2} \quad \text { a.e. }
\end{gathered}
$$

On the other hand, the time derivative of $V_{2}$ is given by

$$
\dot{V}_{2}(t)=h_{\max }|\dot{e}(t)|^{2}-\int_{t-h_{\max }}^{t} \dot{e}(s)^{2} d s .
$$

From (21) and using the sector property of $\eta(\cdot)$, we obtain

$$
\begin{aligned}
& |\dot{e}| \leq(|A|+|K C|+b)|e|+\Psi_{\max }|B||\tilde{\theta}|+|K C| \Gamma \\
& |\dot{e}| \leq\left(\beta_{a}+\beta_{c}+b\right)|e|+\Psi_{\max } \beta_{b}|\tilde{\theta}|+\beta_{c} \Gamma
\end{aligned}
$$

hence

$$
\begin{aligned}
h_{\max }|\dot{e}|^{2} \leq & h_{\max }\left(\beta_{a}+\beta_{c}+b\right)^{2}\left(\lambda_{\min }(P)\right)^{-1} V_{1}(e) \\
& +h_{\max } \beta_{c}^{2} \Gamma^{2}+h_{\max } \mu^{-1} e^{\alpha T}\left(\Psi_{\max } \beta_{b}\right)^{2} V_{3}(t, \tilde{\theta}),
\end{aligned}
$$

where we have used Lemma 1. Next, using the Jensen's inequality, it follows that

$$
h_{\max } \int_{t-h_{\max }}^{t} \dot{e}(s)^{2} d s \geq\left[\int_{t-h(t)}^{t} \dot{e}(s) d s\right]^{2}=\Gamma(t)^{2} .
$$

In summary, using the above inequalities (30)-(34) and re-arranging terms, the total time derivative of $V(t, e, \tilde{\theta})$ along the trajectories of the system (7),

$$
\begin{aligned}
\dot{V}(t, e, \tilde{\theta}) \leq & \left(-\frac{\varepsilon}{\lambda_{\max }(P)}+h_{\max } C_{e}\right) V_{1} \\
& +\left(C_{\theta}+h_{\max } \mu^{-1} e^{\alpha T}\left(\beta_{b} \Psi_{\max }\right)^{2}-\alpha\right) V_{3} \\
& +\left(1-\frac{1}{2 h_{\max }}+h_{\max } \beta_{c}^{2}\right) \Gamma^{2} \quad \text { a.e }
\end{aligned}
$$

where

$$
C_{e}=\left(\lambda_{\min }(P)\right)^{-1}\left(\left(\beta_{a}+\beta_{c}+b\right)^{2}+2\left(\beta_{c} \lambda_{\max }(P)\right)^{2}\right),
$$

$$
C_{\theta}=\mu^{-1} e^{\alpha T}\left(\beta_{b} \Psi_{\max }\right)^{2}\left(\rho^{-1}+\left(\lambda_{\max }(P)\right)^{2}\right) .
$$

Therefore, if $h_{\max }$ satisfies the system of in-equations

we have

$$
\left\{\begin{array}{l}
h_{\max } C_{e}-\frac{\varepsilon}{2 \lambda_{\max }(P)} \leq 0 \\
C_{\theta}+h_{\max } \mu^{-1} e^{\alpha T}\left(\beta_{b} \Psi_{\max }\right)^{2}-\frac{\alpha}{2} \leq 0 \\
1-\frac{1}{2 h_{\max }}+h_{\max } \beta_{c}^{2} \leq 0,
\end{array}\right.
$$

$$
\dot{V} \leq-\frac{\varepsilon}{2 \lambda_{\max }(P)} V_{1}-\frac{\alpha}{2} V_{3} \quad \text { a.e. }
$$

Solving for $h_{\max }$ the system of in-equations (35), we deduce that (36) holds for sufficiently small values of $h_{\max }$ such that

$$
h_{\max } \leq \min \left\{\pi_{a}, \pi_{b}, \pi_{c}\right\}
$$

where $\pi_{a}=C_{e}^{-1}\left(\frac{\varepsilon}{2 \lambda_{\max }(P)}\right), \pi_{c}=\frac{-1+\sqrt{1+2 \beta_{c}^{2}}}{2 \beta_{c}^{2}}$ and $\pi_{b}=\mu e^{-\alpha T}\left(\beta_{b} \Psi_{\max }\right)^{-2}\left(\frac{\alpha}{2}-C_{\theta}\right)$.

Hence, applying the Lyapunov-Krasovskii Stability Theorem (Loria et al. (2005), Theorem 4.1), we conclude that the null solution $(e, \tilde{m})=(0,0)$ (i.e $w=0)$ of the error system (7) is globally asymptotically stable.

\section{NUMERICAL EXAMPLE: A CHAOS-BASED COMMUNICATION SYSTEM WITH A TRANSMISSION TIME-DELAY USING A LUR'E SYSTEM}

We consider a chaotic Lur'e system which consists of two unidirectionally coupled "Chua" systems - see Yalcin et al. 
(2001). A continuous piece-wise constant information $m(t)$ is injected in its dynamics. The output $y=\left[y_{1}, y_{2}\right]^{\top}$ is corrupted by a known and bounded time varying transmission delay $h(t)$ which corresponds to a uniformly distributed piece-wise function generated between lower and upper bounds respectively equal to 0 and 0.03 . That is, the dynamics of the chaotic transmitter is given by

$$
\begin{aligned}
& \dot{x}_{1}=9\left(x_{2}-g\left(x_{1}\right)\right)+(\sin (3 t)+\cos (27 t)) m \\
& \dot{x}_{2}=x_{1}-x_{2}+x_{3} \\
& \dot{x}_{3}=-14.28 x_{2} \\
& \dot{x}_{4}=9\left(x_{2}-g\left(x_{4}\right)\right) \\
& \dot{x}_{5}=x_{4}-x_{5}+x_{6}+0.01\left(x_{5}-x_{2}\right) \\
& \dot{x}_{6}=-14.28 x_{5} \\
& y_{1}=x_{1}(t-h(t)) \\
& y_{2}=x_{4}(t-h(t))
\end{aligned}
$$

with $g\left(x_{i}\right)=\frac{1}{2}\left(\left|x_{i}-1\right|-\left|x_{i}+1\right|\right), i \in\{1,4\}$.

$g(\cdot)$ satisfy the slope restriction property (3) with $b=1$. System (38) is of the form (1), with

$$
\begin{aligned}
& A=\left[\begin{array}{cccccc}
-2.5714 & 9 & 0 & 0 & 0 & 0 \\
1 & -1 & 1 & 0 & 0 & 0 \\
0 & -14.28 & 0 & 0 & 0 & 0 \\
0 & 0 & 0 & -2.5714 & 9 & 0 \\
0 & -0.01 & 0 & 1 & -0.09 & 1 \\
0 & 0 & 0 & 0 & -14.28 & 0
\end{array}\right], \\
& B=\left[\begin{array}{llllll}
1 & 0 & 0 & 0 & 0 & 0
\end{array}\right]^{T}, \\
& F=\left[\begin{array}{cc}
-3.8571 & 0 \\
0 & 0 \\
0 & 0 \\
0 & -3.8571 \\
0 & 0 \\
0 & 0
\end{array}\right] \\
& C=H=\left[\begin{array}{llllll}
1 & 0 & 0 & 0 & 0 & 0 \\
0 & 0 & 0 & 1 & 0 & 0
\end{array}\right],
\end{aligned}
$$

$f(x)=\left[g\left(x_{1}\right) ; g\left(x_{4}\right)\right], \Psi(t)=\sin (3 t)+\cos (27 t)$ and $\theta(t)=m(t)$.

The initial state of the system (38) is set to $x(s)=[-0.2,-0.2,-0.33,0.2,0.9,0.33]^{\top}, \forall s \in[-0.03,0]$. Under these conditions, the transmitter (38) exhibits chaotic behavior (See Figure 1).
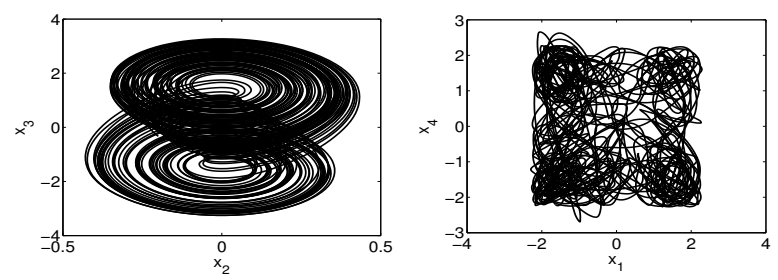

Fig. 1. Attracotrs in the $\left(x_{1}, x_{4}\right)$ plane (right) and $\left(x_{2}, x_{3}\right)$ plane (Left)

The receiver system is given by (6) . Solving the optimization problem given in Remark (3), we have

$$
\begin{gathered}
D=\left[\begin{array}{cccc}
29.8972 & 0 \\
0 & 33.4389
\end{array}\right], \\
P=\left[\begin{array}{ccccccc}
10.43 & 0 & 0 & 0 & 0 & 0 \\
0 & 122.58 & -6.91 & 0 & 0 & 0 \\
0 & -6.91 & 9.11 & 0 & 0 & 0 \\
0 & 0 & 0 & 10.96 & -7.01 & 0.89 \\
0 & 0 & 0 & -7.01 & 74.57 & -7.63 \\
0 & 0 & 0 & 0.89 & -7.63 & 6.32
\end{array}\right],
\end{gathered}
$$

$\varepsilon=8.9250$ and $\varsigma=0$.

We obtain also numeric values of the observer matrices:

$$
K=\left[\begin{array}{cc}
0.1992 & -0.0053 \\
1.8141 & 0 \\
0.5965 & 0 \\
-0.0047 & 1.0278 \\
0.0005 & 2.5823 \\
-0.0002 & 0.3639
\end{array}\right], \quad M=\left[\begin{array}{ll}
10.4397 & 0
\end{array}\right]
$$

$\rho=0.25$ and $\alpha=8$.

The estimated state is initialized at $z(s)=[0,0,0,0,0,0]^{\top}$, $\forall s \in[-0.03,0]$ the equation (6c) is initialized at $\Upsilon(0)=1$ and the estimated message $\hat{m}$ is initialized at $\hat{m}_{0}=1$. The simulation results are as follows. Figure 2 depicts the evolution of the time delay function. Figure 3 illustrates the convergence of the observation errors and finally figure 4 shows that the transmitted information is well recovered by the proposed adaptive observer.

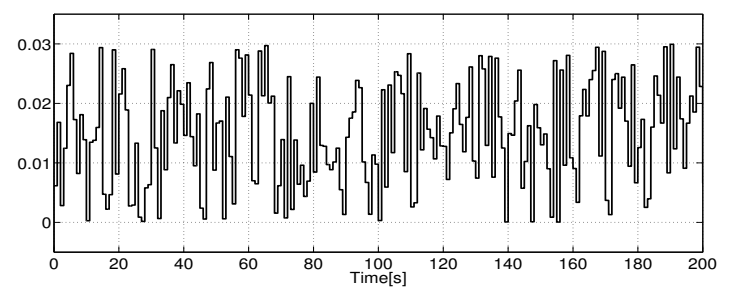

Fig. 2. Evolution of the time delay function $h(t)$

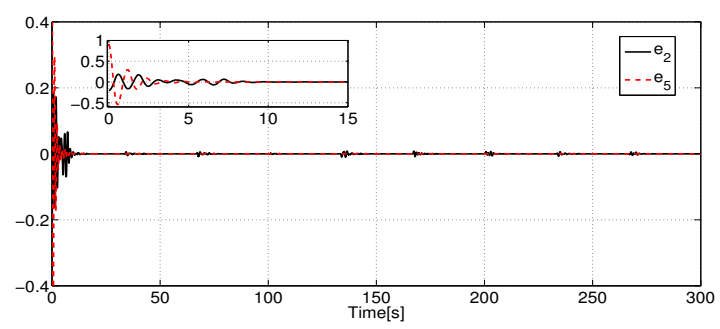

Fig. 3. Estimation errors $e_{2}=x_{2}-z_{2}$ and $e_{5}=x_{5}-z_{5}$ in presence of the time-varying transmission delay

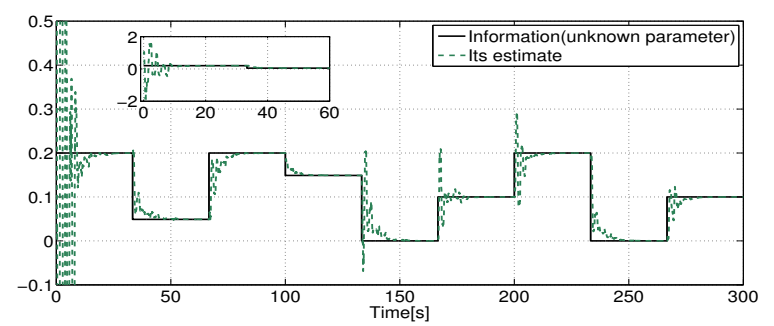

Fig. 4. The transmitted message $m$ and the recovered message $\hat{m}$ in presence of the transmission delay 


\section{CONCLUSION}

In this work, we propose a synchronization method based on adaptive observers for a class of Lur'e systems with slope restricted nonlinearities and delayed outputs. Both state estimation and information reconstruction are shown to be ensured for sufficiently small upper bounds of the delay if a condition of persistent excitation is satisfied. The design matrices of the observer are obtained after solving a convex optimization problem. For illustration, the approach was evaluated through a chaotic-based communication system subject to a transmission time-delay. In future works, we will investigate the case of long timedelays using cascade observers.

\section{REFERENCES}

Ahmed-Ali, T., Cherrier, E., and Msaad, M. (2009). Cascade high gain observers for nonlinear systems with delayed output. In Proc. 48th. IEEE Conf. Decision Contr., 8226 -8231. Shanghai, China.

Assche, V.V., Ahmed-Ali, T., Hann, C.A.B., and Lamnabhi-Lagarrigue, F. (2011). High gain observer design for nonlinear systems with time varying delayed measurements. In 18th IFAC World congress, 692-696. Milano, Italia.

Cacace, F., Germani, A., and Manes, C. (2010). An observer for a class of nonlinear systems with time varying observation delay. Sys. $\&$ Control Letters, 59, $305-312$.

Cao, J., Li, H.X., and Ho, D.W.C. (2005). Synchronization criteria of lure systems with time-delay feedback control. Chaos, Solitons and Fractals, 23, 1285-1298.

Dimassi, H. and Loria, A. (2011). Adaptive unknown input observers-based synchronization of chaotic systems for telecommunicaion. IEEE Trans. on Circ. Syst. I: Regular Papers, 58, 800-812.

Feki, M. (2003). An adaptive chaos synchronization scheme applied to secure communication. Chaos, Solitons and Fractals, 18, 141-148.

Germani, A., Manes, C., and Pepe, P. (2000). A new approachto state observation of nonlinear systems with delayed output. IEEE Trans. on Automat. Contr., 47 (1), 96-101.

Han, Q.L. (2007a). New delay-dependent synchronization criteria for lur'e systems using time delay feedback control. Phys. Lett. A, 360, 563-569.

Han, Q.L. (2007b). On designing time-varying delay feedback controllers for master-slave synchronization of lur'e systems. IEEE Trans. on Circ. Syst. I: Regular Papers, 54, 1573-1583.

Lee, S.M., Choi, S.J., Ji, D.H., Park, J.H., and C.Won, S. (2010). Synchronization for chaotic lure systems with sector-restricted nonlinearities via delayed feedback control. Nonlinear Dyn, 59, 277-288.

Loria, A., Lamnabhi-Lagarrigue, F., and Panteley, E. (2005). Advanced topics in control systems theory. Series Lecture Notes in Control and Information Science, Springer Verlag, London, ISBN: 1-84628-313-2.

Loria, A., Panteley, E., and Zavala, A. (2009). Adaptive observers with persistency of excitation for synchronization of chaotic systems. IEEE Trans. on Circ. Syst. I: Regular Papers, 56(12), 2703-2716.
Nijmeijer, H. and Mareels, I. (1997). An observer looks at synchronization. IEEE Trans. on Circ. Syst. I: Fundamental Theory and Applications, 44 (10), 882890.

Pan, Y.J. (2008). robust observer-based output tracking control of nonlinear systems with sensor measurement delays. In 17th IFAC World congress, 2785-2790. Seoul, Korea.

Yalcin, M.E., Suykens, J.A.K., and Vandewalle, J. (2001). Master-slave synchronization of lure systems with timedelay. Int. J. of Bifurcat. and Chaos, 11 (6), 1707-1722.

\section{Appendix A. PROOF OF LEMMA 1}

Let $\Upsilon(t)$ the solution of the equation (6c). We have

$$
\begin{aligned}
\frac{d}{d t}\left(e^{\alpha t} \Upsilon(t)\right) & =e^{\alpha t}(\dot{\Upsilon}(t)+\alpha \Upsilon(t)) \\
& =e^{\alpha t} \Psi(t)^{T} B^{T} B \Psi(t)
\end{aligned}
$$

Integrating the previous equation from $t_{0}$ to $t+T$,

$$
\begin{aligned}
e^{\alpha(t+T)} \Upsilon(t+T) & =e^{\alpha t_{0}} \Upsilon\left(t_{0}\right)+\int_{t_{0}}^{t+T} e^{\alpha s} \Psi(s)^{T} B^{T} B \Psi(s) d s \\
& \geq \int_{t_{0}}^{t+T} e^{\alpha s} \Psi(s)^{T} B^{T} B \Psi(s) d s
\end{aligned}
$$

Multiplying on both sides by $e^{-\alpha(t+T)}$, we obtain

$$
\begin{aligned}
\Upsilon(t+T) & \geq \int_{t_{0}}^{t+T} e^{\alpha(s-t-T)} \Psi(s)^{T} B^{T} B \Psi(s) d s . \\
& \geq \int_{t}^{t+T} e^{\alpha(s-t-T)} \Psi(s)^{T} B^{T} B \Psi(s) d s .
\end{aligned}
$$

For $t \leq s \leq t+T, e^{-\alpha T} \leq e^{\alpha(s-t-T)} \leq 1$, hence

$$
\Upsilon(t+T) \geq e^{-\alpha T} \int_{t}^{t+T} \Psi(s)^{T} B^{T} B \Psi(s) d s .
$$

Finally, using equation (12), $\forall t \geq t_{0}+T$, we obtain

$$
\Upsilon(t) \geq \mu e^{-\alpha T} I
$$

